\title{
SYSTEM FOR ACTIVE VIDEO OBSERVATION OVER THE INTERNET
}

\author{
Borut Batagelj, Peter Peer, Franc Solina \\ University of Ljubljana \\ Faculty of Computer and Information Science \\ Computer Vision Laboratory \\ Tržaška 25, 1000 Ljubljana, Slovenia \\ \{borut.batagelj, peter.peer, franc.solina\}@fri.uni-lj.si
}

\begin{abstract}
We designed a system with intuitive user interface for remote camera control and image-based queries over the Internet. While searching for present solutions we realized the importance of a well-designed user interface. We developed a system, which enables remote observation and remote control of the JVC network camera over the Internet. User interface is based on the combination between live video and static panoramic view of remote location. It provides a complete overview of a remote location and significantly simplifies the control over the Internet. By interactively moving a rectangular frame in the panoramic picture the user locally selects the new direction of the camera. Visual summaries of activities at the observed location can be generated as well as custom queries with a simple user interface over the Internet.
\end{abstract}

Keywords: user interface, live video, panoramic view, network camera control

\section{INTRODUCTION}

Live video transmission over the Internet and interactivity are becoming more and more popular. On the World Wide Web we can find hundreds of cameras showing live images from selected locations [8]. Video can give us information that static images can not. The image transmission speed is increasing with further development of technology and Internet infrastructure. Therefore, the course of development will focus on intelligent control systems, which will enable controlled camera movements and image database queries over the Internet. Previously, Prihavec and Solina developed the system named Internet Video Server [6]. It was based on the analog camera that was attached to a special pan-tilt unit. Prihavec and Solina also developed a special software which enables remote control of the pan-tilt unit over the internet and a user interface, based on the panoramic view [5]. The purpose of our re-implementation of the system was to improve it so that it would work with a JVC network camera [1] and upgrade our system with the database feature [2], in which we store the images on request or automatically when an external event occurs. Image queries are then possible over the Internet.

We use the JVC network camera VN-C3WU which is digital camera with the pan/tilt unit. It contains a computer and a compression chip. The computer is small and specialized for network applications. The JVC network camera has its own IP address. It is connected to the network as a network device and it has build-in web server. It has $10 \times$ optical zoom and input/output alarm capability. 
When using present solutions for video observation over the internet which feature live video streaming and navigation with the arrow-based user interface ${ }^{1}$, we noticed some problems. Because of the slow and irregular responses to the users requests, the system does not seem to be very predictable. The second problem with such a user interface is even more perceivable. If the focal length of the lens is large one can easily loose the notion where the camera is pointing at. When moving the camera step by step, we can not predict the exact final position of it, nor do we know what are the relations between the object in the image and other objects on the scene. We can solve all these problems with an improved interface, which includes panoramic view of the whole observed environment.

In Section 2 we describe the system and learn about how different parts are connected to each other. In Section 3 we explain how the panoramic views are generated. The panoramic image is then used as one part of the user interface, which is presented in Section 4. Section 5 describes the application that is used to make visual summaries. Conclusions in Section 6 give a short summary of presented work and some practical ideas where to use such a system.

\section{ARCHITECTURE}

The system consists of the main server, client, database and the network camera (Fig. 1).

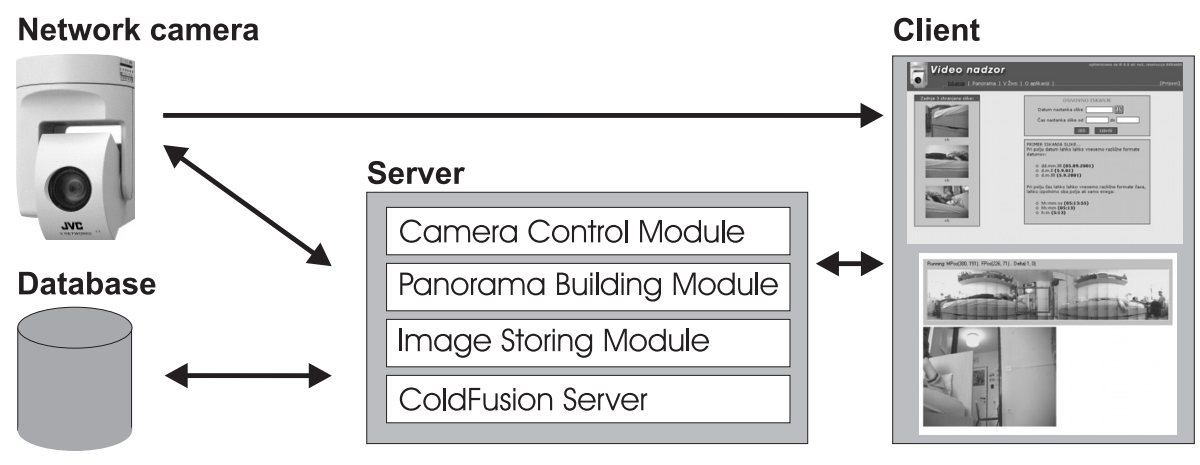

Fig. 1: System architecture.

The central part of our system is the main server, which is built of four modules:

- Camera control module. Its main tasks are serving the requests that come from clients and controlling the camera's pan-tilt unit by executing camera's CGI programs.

- Module for generation of panoramic view. At system startup module moves the camera in all directions and receives the images. At the end it transfers the panoramic image to the user.

- Module for storing images. This module is called every few minutes, but it could also be called on special occasions, like when the motion is detected.

\footnotetext{
${ }^{1}$ Navigation is possible by clicking the buttons which issue the command to the pan/tilt unit to move the camera in the predefined direction.
} 
- ColdFusion server. It generates visual summaries on client request.

Database stores information about the camera and the image parameters. Network camera has its own web server which transmits live video to the clients and separate images to the main server. We control the camera with the user interface program written in Java. Image database queries are requested through ColdFusion application, i.e. databasedriven web pages.

\section{PANORAMIC VIEWS}

We can generate panoramic images with a special omnidirectional sensors or a conventional image-based system. In our system we rotate the camera over the scene, assuming that the camera is rotating around its optical center, and integrate overlapping images into panoramic image. With this technique, large panoramic images of arbitrary shape and detail can be created [7]. In our case, the camera direction information is available and we know the horizontal and vertical view angles of the camera lens. By knowing these, we can perform image composition automatically without the need to calculate the relative position between two successive images from the images themselves. Using the camera's pan-tilt unit position information, we know the precise position of the captured image within the whole panoramic image. To get a rectangular panoramic image the individual images must be transformed from spherical to cylindrical coordinates. The panoramic images obtained in this way have a uniform resolution.

We are assuming that the optical center of the camera is located in the center of a sphere and the camera can observe the inner surface of the sphere. From the camera's point of view every scene can be represented as an image mapped onto the inner surface of the sphere - a spherical panoramic image. Next, the spherical panoramic image must be transformed to fit onto the surface of a cylinder to produce the cylindrical panoramic image. Fig. 2 shows how this transformation is performed.

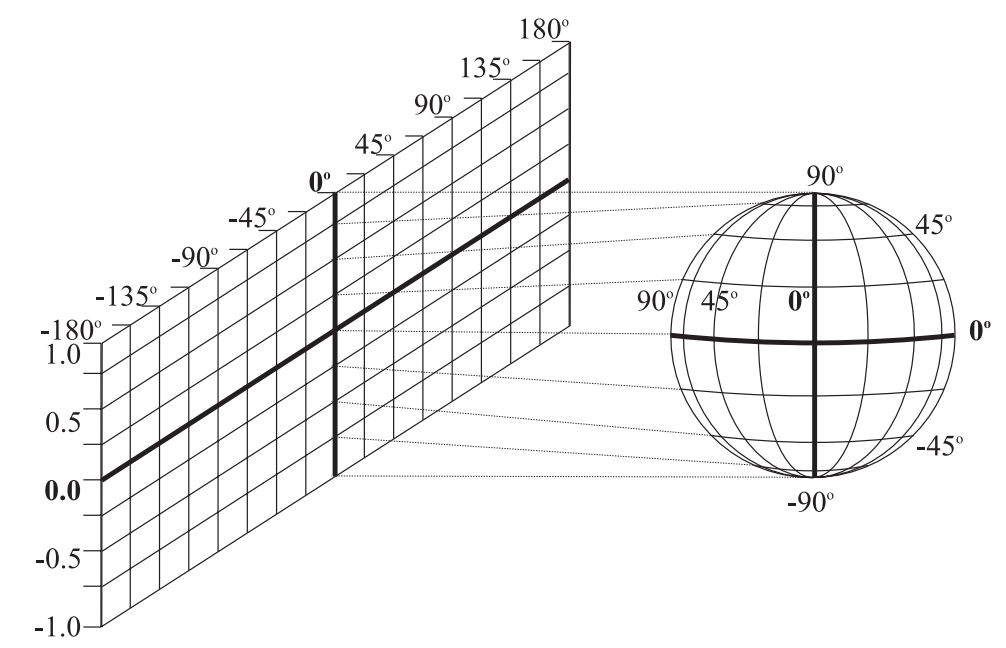

Fig. 2: Projection of the spherical images onto the cylindrical surface.

The transformation consists of three steps $[1,3,5]$ : 
1. Mapping of an image $I$ onto the projection plane (Cartesian coordinates) $\left(I \rightarrow I_{p}\right)$

2. Transformation into spherical coordinates $\left(I_{p} \rightarrow I_{s}\right)$

3. Transformation into cylindrical coordinates $\left(I_{s} \rightarrow I_{c}\right)$

Individual image $I$ is defined as a matrix of pixel values $I(i, j)$ where $i \in\left[-\frac{W}{2}, \frac{W}{2}\right]$ and $j \in\left[-\frac{H}{2}, \frac{H}{2}\right] . W$ and $H$ are image width and image height.

In spherical coordinates every point on the surface of the sphere can be represented as $I_{s}(\varphi, \vartheta)$, where $\varphi$ is the angle in the horizontal direction $(\varphi \in[-\pi, \pi])$ and $\vartheta$ is the angle in the vertical direction measured from the sphere horizon $\left(\vartheta \in\left[-\frac{\pi}{2}, \frac{\pi}{2}\right]\right)$.

Every point on the cylinder surface can be represented as $I_{c}(\psi, v)$, where $\psi$ is the angle in horizontal direction $(\varphi \in[-\pi, \pi])$ and $v$ is the elevation.

\section{INTUITIVE CAMERA CONTROL USER INTERFACE}

User interface for camera control (Fig. 3) is a combination of a static panoramic view and live image stream received from the camera.

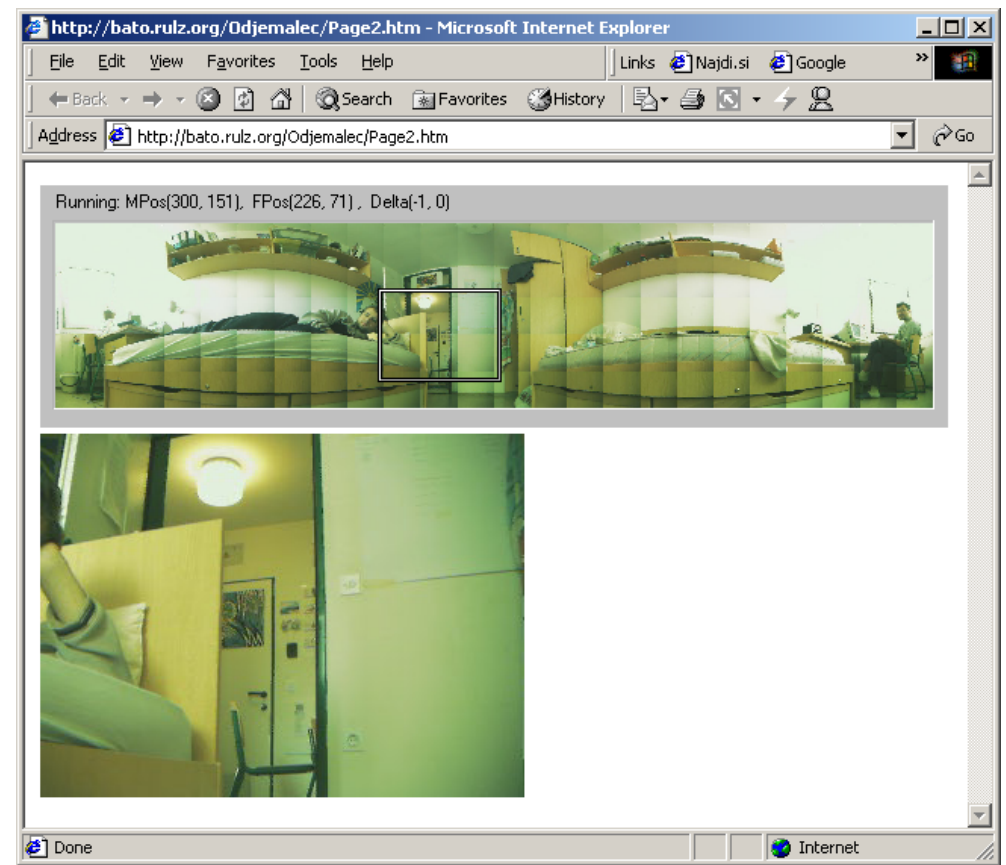

Fig. 3: Intuitive user interface for camera control.

At the system startup the panoramic image is first generated by scanning the complete surrounding of the camera. When a user starts interacting with the system he or she sees the whole panoramic image. A rectangular frame in the panoramic image indicates the current direction of the camera. The user can move the attention window with a mouse and in this way controls the direction of the camera. From the position of the attention window within the panoramic image the physical coordinates of the next camera direction are computed and appropriate command is issued to the camera's pan-tilt unit. Before 
the camera moves the last live image is superimposed on the corresponding position in the panoramic view.

The whole interaction with the system is carried out through the attention window. Moving this attention window results in the camera movement. With changing the size of the attention window, we can change the zoom of the camera. At any time, only one user can control the camera, since allowing more than one user to interact with it could be confusing. To allow a fair time-sharing every user can control the camera for a few minutes and after that the control is passed to the next user waiting in the queue.

\section{VISUAL SUMMARY REPORT}

When using the system, we sometimes wish to see what was happening at a remote location in the past. To enable this kind of functionality, we extended our system with a database feature $[2,4]$. In the database we are saving live video frames from remote location with a time stamp and some camera information like pan and tilt position, zoom position, compression rate, image size, etc.

We are using ColdFusion web application server for the generation of visual summary reports. ColdFusion enables queries over the internet. It uses ColdFusion Markup Language (CFML), a set of special tags that are typically placed inside HTML pages and interpreted by the ColdFusion Server. With the ColdFusion application users can request (Fig. 4) and see the results of different kinds of customized queries within their favorite web browser. The results can be presented as separate pictures, a movie or in the panoramic image.

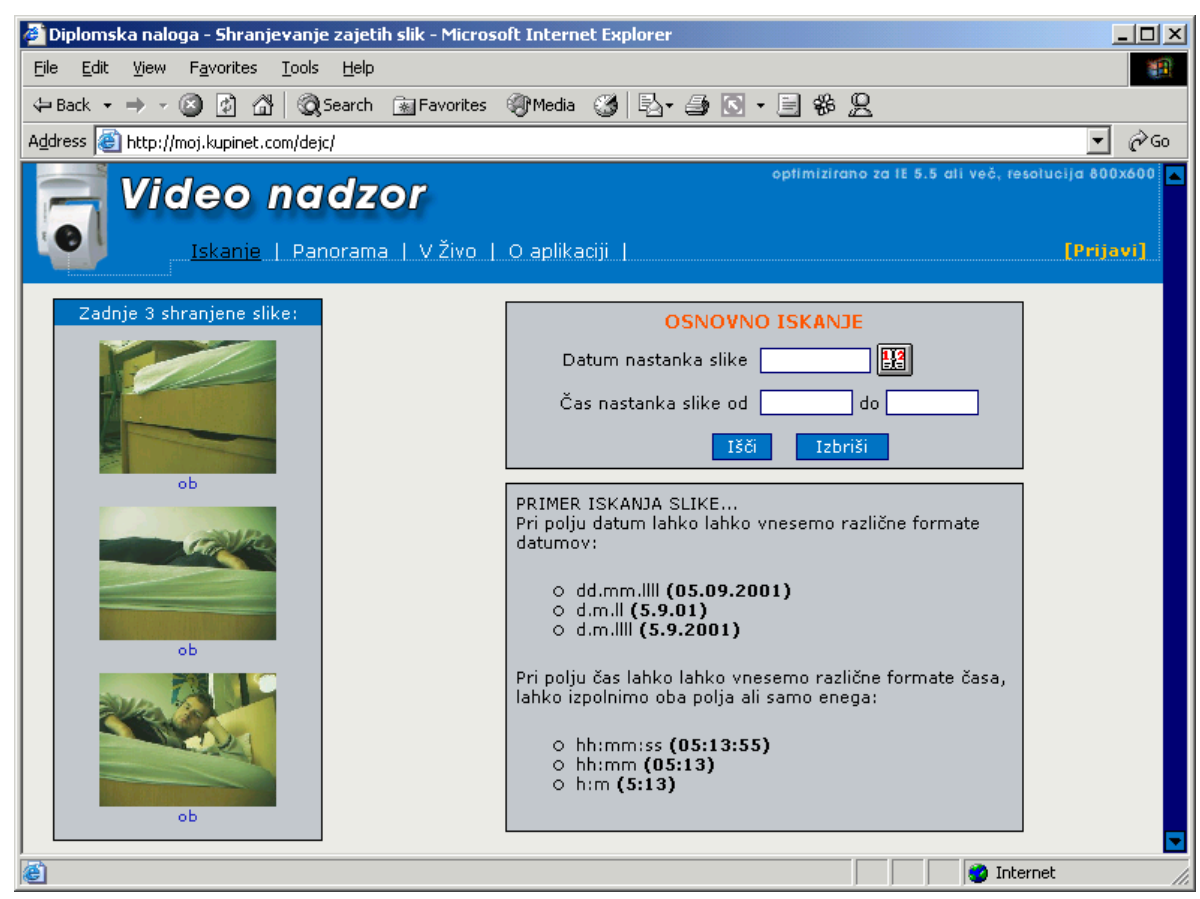

Fig. 4: User interface for quering the database. 


\section{CONCLUSIONS}

Our system extends existing systems for remote camera control over the Internet. With a panoramic image of the whole environment the observer gains a better understanding of the observed location and a more intuitive control of the camera. Database allows us to make visual summaries of activities at the observed location. Video-conferencing and remote surveillance are examples of applications that would certainly benefit from presented ideas.

\section{REFERENCES}

[1] B. Batagelj, System for active video observation over the internet, Diploma Thesis, Faculty of Computer and Information Science, Ljubljana, Slovenia, 2001 (in Slovenian language)

[2] D. Meštrič, Web-based quering application for video monitoring system, Diploma Thesis, Faculty of Computer and Information Science, Ljubljana, Slovenia, 2001 (in Slovenian language)

[3] Theo Moons, A guided tour through Multiview Relations, Proc. of SMILE'98 workshop, LNCS 1506, 1998, 304-346

[4] P. Peer, F. Solina, SecurityAgent, Electrotechnical and Computer Science Conference ERK'01, Portorož, Slovenia, 2001, 119-122 (in Slovenian language)

[5] B. Prihavec, F. Solina, User Interface for Video Observation over the Internet, Journal of Network and Computer Applications, 21, 1998, 219-237

[6] B. Prihavec, F. Solina, Sending live video over Internet, Workshop of the Austrian Association for Pattern Recognition (ÖAGM/AAPR), Hallstatt, Austria, 1997, 299303

[7] R. Szeliski, Video Mosaics for Virtual Environments, IEEE Computer Graphics and Applications, 16(2), 1996, 22-30

[8] EarthCam - Webcam Network, http://www.earthcam.com

\section{Acknowledgments}

This work was supported by the Ministry of Education, Science and Sport of Republic of Slovenia (Research program 1539-506). 